\title{
Optimal Clutch Control during Launch on the One-way Clutch Assistant Transmission
}

\author{
Yang Yong ${ }^{1, \text { a }}$, Zhao Kegang ${ }^{1}$, Li Gang ${ }^{2}$ and Wei Jianjun ${ }^{2}$ \\ ${ }^{1}$ South China University of Technology, Guangzhou, Guangdong, 510640, China \\ ${ }^{2}$ Automotive Engineering Institute, Guangzhou Automobile Group Corporation, Ltd., Guangzhou, \\ Guangdong, 510640, China \\ atc-yangyong@hotmail.com
}

Keywords: OCT; Launch; Optimal clutch control

\begin{abstract}
A novel type of automatic transmission is presented in this paper. The transmission consists of a fiction clutch and a one-way clutch, which is named as One-way Clutch Assistant Transmission. The working principle of this type of transmission is shifting automatically by automatic disengagement and engagement characters of the one-way clutch. The structure of the transmission is similar as Manual Transmission, when it is in the condition of launch. A simplified dynamic model of launch is built in this paper. The clutch controller based on optimal control method is also proposed. Simulations and tests are carried out. The results of both simulation and test prove the dynamic model and control strategy build in the paper can be used to study the dynamic characters of the OCT during launch.
\end{abstract}

\section{Introduction}

Traditional Automated Manuel Transmissions (AMTs) have the efficiency as high as that on Manuel Transmissions (MTs). One clutch is used to transmit the engine torque on the AMT, which is similar as the MT. The clutch is forced to disengage and engage by actuator of either electric-drive or hydraulic-drive during processes of launch and shifts. A clutch controller is always designed to control the clutch during launch process. Closed-loop technique is employed to build the clutch controller. Closed-loop methods are mainly used to control the clutch during launch: (a) optimal control method, (b) fuzzy control method, and (c) sliding-mode control method.

Qin Datong used linear quadratic optimal control method to achieve the optimum launch control of the clutch, in which the jerk is converted to one of the restrain conditions, and the minimization of sliding friction work of the clutches is set as optimal target in control [1]. Tongli Lu found compromise between the friction loss, the shock intensity, the engine torque and the engine angular acceleration to deduce the optimal engagement laws. He also used a fuzzy control theory to calculate the driver's starting intentions [2]. Joachim Horn provided an electro-hydraulic clutch control system. The controller was based on the flatness approach. Nonlinear feed forward control was employed. Experiments were conducted by this controller, provided an accurate trajectory tracking of the clutch position [3]. Xingyong Song designed a sliding-mode controller to achieve robust pressure control while avoiding the chattering effect of the clutch during launch [4]. The minimum value principle is introduced to achieve an optimal clutch engagement compromised in average shock intensity and friction wear $[5,6,7,8]$.

The disadvantage of the AMT is the traction interruption during shifts, which makes the passengers uncomfortable. A one-way clutch is used to transmit engine torque during shifts by a novel type of transmission presented in this paper. The controller is designed based on optimal control method to control the clutch during launch. 


\section{Structure Layout}

One-way Clutch Shift-assistant Transmission (OCT) is a novel type of automatic transmission which shifts without traction interruption. The working principle of this type of transmission is shifting automatically by the automatic disengagement and engagement characters of the one-way clutch. The idea behind this transmission concept is to fix two manual gearboxes together as a whole. One half of the OCT, which consists of a friction clutch, mainly transmits power from the engine. The other half, which consists of a one-way clutch, is used as power assistance during shifts. Each of the two halves of the OCT is connected to the engine separately. The structure layout of the OCT is shown in Fig.1.
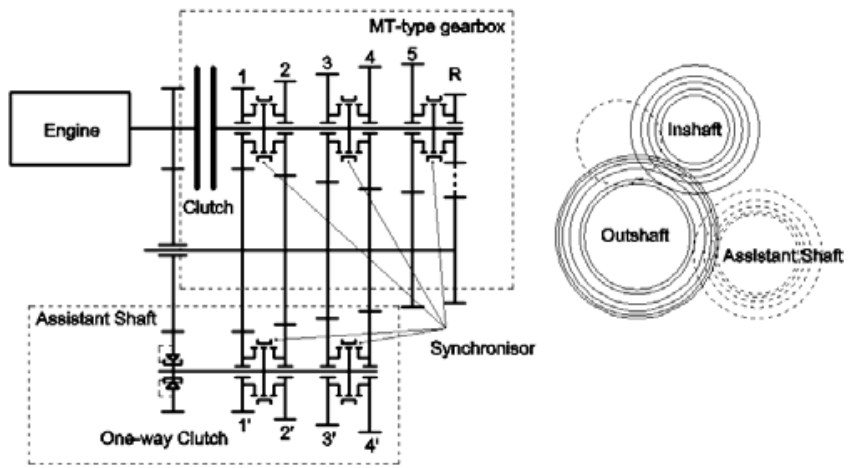

Fig. 1 Structure layout of the One-way Clutch Assistant Transmission

The OCT can be divided into two parts: MT-type gearbox and assistant input shaft. When the transmission is in launch process, it seems like an AMT. Because the synchronizer of gear $1^{\text {st }}$ is engaged before launch, and the fiction clutch is controlled to engage by the clutch controller. Synchronizers of other gears are disengaged during launch.

\section{Dynamic Powertrain Model}

A dynamic model of powertrain consisting of elements which transmit the engine torque to wheels is developed, as shown in Fig.2. The synchronizer of gear $1^{\text {st }}$ is ignored, because all parts of the synchronizer are connected as a static component when it is engaged. It can be seen as massspring-damper systems for both of input shaft and output shaft. Other components of the transmission are ignored because of the slight influence of these parts to the system in the launch condition.

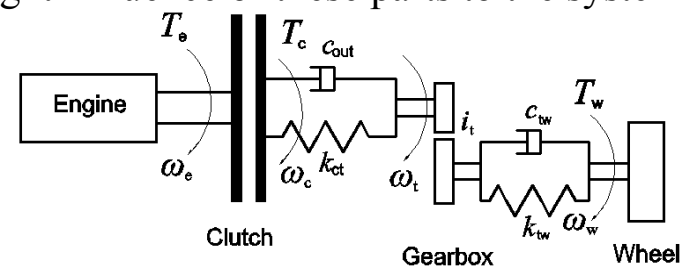

Fig. 2 Dynamic model of the powertrain in launch condition

Dynamic functions expressing the model depicted in Fig.2 are as following:

$$
\begin{gathered}
I_{\mathrm{e}} \dot{\omega}_{\mathrm{e}}=T_{\mathrm{e}}-T_{\mathrm{c}} \\
I_{\mathrm{c}} \dot{\omega}_{\mathrm{c}}=T_{\mathrm{c}}-k_{\mathrm{ct}} \Delta \theta_{\mathrm{ct}}-c_{\mathrm{ct}}\left(\omega_{\mathrm{c}}-\omega_{\mathrm{t}}\right) \\
I_{\mathrm{t}} \dot{\omega}_{\mathrm{t}}=k_{\mathrm{ct}} \Delta \theta_{\mathrm{ct}}+c_{\mathrm{ct}}\left(\omega_{\mathrm{c}}-\omega_{\mathrm{t}}\right)-\frac{1}{i_{\mathrm{t}}}\left[k_{\mathrm{tw}} \Delta \theta_{\mathrm{tw}}+c_{\mathrm{tw}}\left(\frac{\omega_{\mathrm{t}}}{i_{\mathrm{t}}}-\omega_{\mathrm{w}}\right)\right] \\
I_{\mathrm{w}} \dot{\omega}_{\mathrm{w}}=k_{\mathrm{tw}} \Delta \theta_{\mathrm{tw}}+c_{\mathrm{tw}}\left(\frac{\omega_{\mathrm{t}}}{i_{\mathrm{t}}}-\omega_{\mathrm{w}}\right)-T_{\mathrm{W}}
\end{gathered}
$$


Where $I_{\mathrm{e}}$ is mass moment of inertia of the rotational elements of the engine, $I_{\mathrm{c}}$ is mass moment of inertia of the driven part of the clutch, $I_{\mathrm{t}}$ is equivalent mass moment of inertia of the input shafts, output shaft and synchronizers on the main input shaft, $I_{\mathrm{w}}$ is equivalent mass moment of inertia of the wheels and the vehicle. $T_{\mathrm{e}}$ is the output torque of the engine, $T_{\mathrm{c}}$ is the torque transmitted through the clutch, $T_{\mathrm{w}}$ is the output torque of the wheels. $\omega_{\mathrm{e}}$ is rotational speed of the crankshaft of the engine, $\omega_{\mathrm{c}}$ is rotational speed of the driven part of the clutch, $\omega_{\mathrm{t}}$ is rotational speed of the input shaft, $\omega_{\mathrm{w}}$ is rotational speed of the wheel. $\Delta \theta_{\mathrm{ct}}$ is rotational angle difference between the driven part of the clutch and the drive gear of gear $1^{\mathrm{st}}, \Delta \theta_{\mathrm{tw}}$ is rotational angle difference between the driven gear of gear $1^{\text {st }}$ and the output shaft. $k_{\mathrm{ct}}$ is stiffness of the input shaft, $k_{\mathrm{tw}}$ is stiffness of the output shaft and the axle shafts of the vehicle. $c_{\mathrm{ct}}$ is damp of the input shaft, $c_{\mathrm{tw}}$ is damp of the output shaft and the axle shafts of the vehicle. $i_{\mathrm{t}}$ is total gear ratio of gear $1 \mathrm{st}$, which contains the gear ratio of gear box $\left(i_{\mathrm{g}}\right)$ and the differential gear ratio $\left(i_{0}\right)$.

$$
i_{\mathrm{t}}=i_{\mathrm{g}} \cdot i_{0}
$$

The clutch is locked at the end of the engagement. The speed of the drive and driven parts of the clutch is the same, which means the clutch rotates as a whole component. According to equations (1) and (2), expression is given by:

$$
\left(I_{\mathrm{e}}+I_{\mathrm{c}}\right) \dot{\omega}_{\mathrm{e}}=T_{\mathrm{e}}-k_{\mathrm{ct}} \Delta \theta_{\mathrm{ct}}-c_{\mathrm{ct}}\left(\omega_{\mathrm{c}}-\omega_{\mathrm{t}}\right)
$$

When the clutch is locked, the friction disc is connected to the input shaft, which means $\omega_{\mathrm{c}}=\omega_{\mathrm{t}}$, equations (2) and (3) can be expressed as:

$$
\left(I_{\mathrm{c}}+I_{\mathrm{t}}\right) \dot{\omega}_{\mathrm{c}}=T_{\mathrm{c}}-\frac{1}{i_{\mathrm{t}}}\left[k_{\mathrm{tw}} \Delta \theta_{\mathrm{cw}}+c_{\mathrm{tw}}\left(\frac{\omega_{\mathrm{c}}}{i_{\mathrm{t}}}-\omega_{\mathrm{w}}\right)\right]
$$

\section{Clutch Controller Design}

Jerk and sliding friction work are the most two important characters to measure whether the engagement of the clutch is quick enough or not. The objective function is built based on jerk and slipping energy calculated as following:

Jerk is the differential of the longitudinal acceleration of the vehicle, shown as the function (8). The acceleration is calculated by the engine torque transmitted to the wheels.

$$
j=\frac{\mathrm{d} a}{\mathrm{~d} t}=\frac{1}{\delta M r_{\mathrm{r}}}\left(\left(i_{\mathrm{g}} i_{0} \eta_{\mathrm{T}}\right) \frac{\mathrm{d} T_{\mathrm{e}}}{\mathrm{d} t}\right)=\frac{i_{\mathrm{g}} i_{0} \eta_{\mathrm{T}}}{\delta M r_{\mathrm{r}}} \cdot \frac{\mathrm{d} T_{\mathrm{e}}}{\mathrm{d} t}
$$

Where $a$ is acceleration of the vehicle, $M$ is the mass of the vehicle, $\delta$ is the equivalent rotational inertia of the gear train, $r_{\mathrm{r}}$ the equivalent radius of the wheel, $\eta_{\mathrm{T}}$ is the efficiency of the gear box.

Sliding friction work $\left(W_{\mathrm{c}}\right)$ is used to measure the energy cost, shown as function (9):

$$
W_{\mathrm{c}}=\left[\int_{0}^{t_{\mathrm{c}_{1}}} T_{\mathrm{c}}(t) \omega_{\mathrm{c}}(t) \mathrm{d} t+\int_{t_{\mathrm{c}_{1}}}^{t_{\mathrm{c}_{2}}} T_{\mathrm{c}}(t)\left(\omega_{\mathrm{e}}(t)-\omega_{\mathrm{c}}(t)\right) \mathrm{d} t\right]
$$

Where $t_{\mathrm{c} 1}$ is the time the vehicle begins to move, $t_{\mathrm{c} 2}$ is the start time of the synchronization of the engine and the clutch.

The objective function is built as following:

$$
\min f=\alpha \cdot j+\beta \cdot W_{\mathrm{c}}
$$

Where $\alpha$ and $\beta$ are the weighting factors of jerk and friction work. The addition of $\alpha$ and $\beta$ is 1 .

There are five working conditions with different factors group of $\alpha$ and $\beta$ (shown in Tab. 1). Where the lowest engagement of the clutch is controlled as group A, and the fastest engagement of the clutch is controlled as group E. 
Tab.1 Weighting factors group of $\alpha$ and $\beta$

\begin{tabular}{cccccc}
\hline & $\mathrm{A}$ & $\mathrm{B}$ & $\mathrm{C}$ & $\mathrm{D}$ & $\mathrm{E}$ \\
\hline$\alpha$ & 1 & 0.6 & 0.5 & 0.4 & 0 \\
$\beta$ & 0 & 0.4 & 0.5 & 0.6 & 1 \\
\hline
\end{tabular}

\section{Simulation Results}

Simulation based on the dynamic models and optimal control method is taken on the Matlab/ Simulink platform. The engine throttle of $10 \%$ and $100 \%$ is taken into account when the vehicle is on the level road. The mode of the clutch is set to group A and $\mathrm{E}$ which simulate the conditions of the slowest and fastest engagement of the clutch. The weighting factors of $\alpha$ and $\beta$ are set to $(1,0)$ and $(0,1)$ during the two conditions. The engine speed and the speed of the output shaft are used as the input signal of the clutch controller. As the synchronizer of gear 1st is engaged during the engagement of the clutch, the speed of the output shaft is related to the speed of the driven part of the clutch.

The displacement of the fiction disc of the clutch is depicted in Fig.3. The clutch engages slowly when throttle is $10 \%$, because jerk is 1 in this condition (Fig. 3 (a)). The fiction clutch moves quickly during $1 \mathrm{~s}$ and $1.3 \mathrm{~s}$. Then its speed comes to slow between $1.3 \mathrm{~s}$ and $1.9 \mathrm{~s}$. The clutch engages fast again at the end of the engagement. The displacement of the clutch working in throttle of $100 \%$ is presented in Fig.3 (b), where engagement of the clutch takes less time than that in Fig.3 (a).

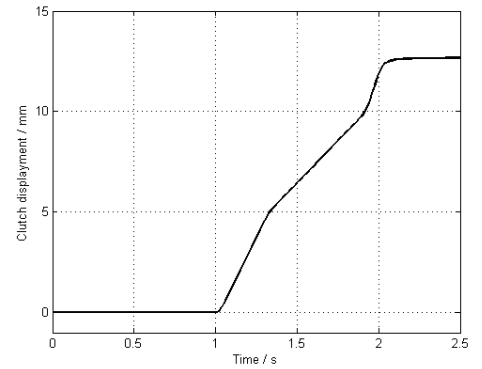

(a) Throttle of the engine is $10 \%$

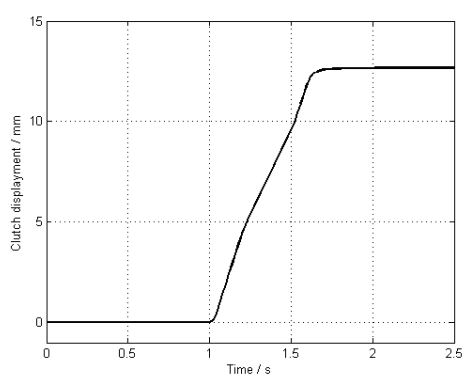

(b) Throttle of the engine is $100 \%$

Fig.3 Simulation results of displacement of the clutch

The clutch rotational speed is depicted in Fig.4. Jerk in the two conditions are depicted in Fig.5.

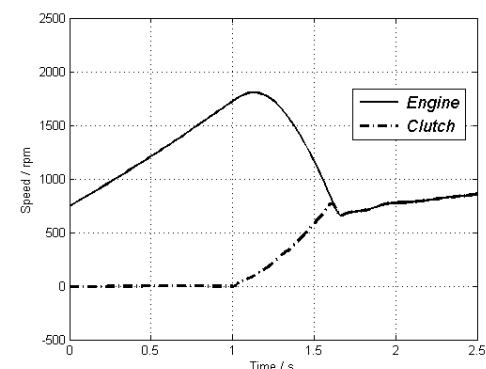

(a) Throttle of the engine is $10 \%$

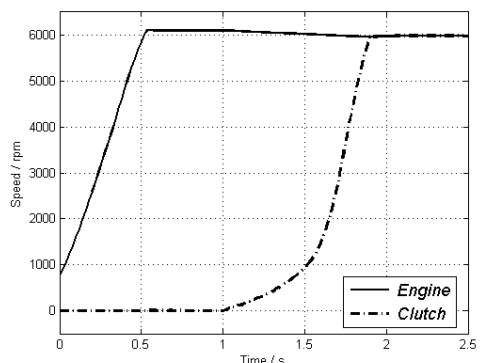

(b) Throttle of the engine is $100 \%$

Fig.4 Simulation results of rotational speed of the clutch and the engine 


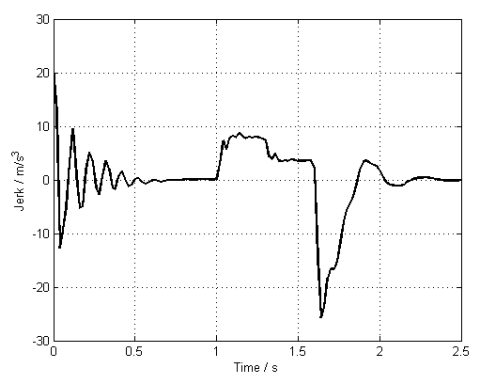

(a) Throttle of the engine is $10 \%$

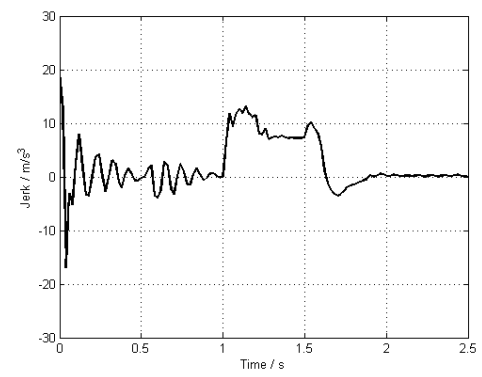

(b) Throttle of the engine is $100 \%$

Fig.5 Simulation results of jerk of the Vehicle

The simulation results show the differences between two control logic while one is the fast launch and the other is slow launch.

\section{Test results}

Test Rig. A bench test consists of a diesel engine, a 5-speed manual gearbox, a clutch actuator, shifts actuators and a control unit is composed in this paper. Load is providing by a water-power dynamometer. Sensors for the test include engine speed sensor, input shaft speed sensor, output shaft speed sensor, speed sensor of the water-power dynamometer, position sensor of the clutch, position sensors of the gears and the accelerator pedal. The working principle of the test bench is shown in Fig.6. Equipment and parameters of the test rig are reported in Tab. 2.

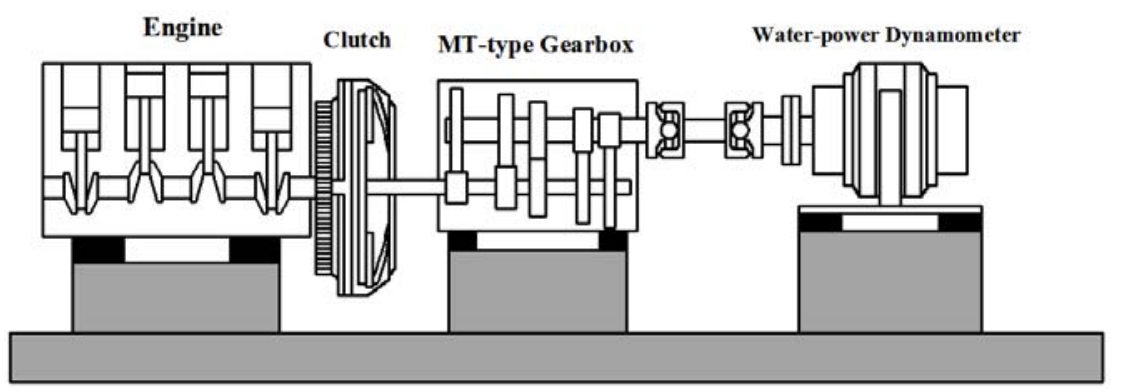

Fig.6 Working principle of the test bench

Tab. 2 Equipment and parameters

\begin{tabular}{cc}
\hline Items & Type or Parameters \\
\hline Engine & IVECO, 814043, 2.8 [L], Diesel engine \\
gearbox & Dongfeng, 17GQ-00030, 5-speed gearbox \\
Load & Taian, 12PSB-HT, Water power dynamometer \\
accelerator pedal & Nissan, 3000 [H] \\
position sensor of the clutch & Rotary slide rheostat, accuracy 0.1\% \\
Throttle sensor & Rotary slide rheostat, accuracy $0.1 \%$ \\
gear position sensor & Linear slide rheostat, accuracy $0.1 \%$ \\
engine speed sensor & magneto-electrical, Voltage peak: $5.4[\mathrm{~V}]$ \\
input speed sensor of gearbox & Hall, Voltage peak: $12[\mathrm{~V}]$ \\
output speed sensor of gearbox & Hall, Voltage peak: $12[\mathrm{~V}]$ \\
\hline
\end{tabular}

Results. The clutch is forced to engage and disengage by a DC motor. The motor's current and voltage are observed during action of the clutch. The position of the driven part of the clutch is calculated by the clutch controller. The current and voltage of the motor during disengagement and engagement of the clutch is depicted in Fig.7. Fig.7 (a) shows the current raises up to 7(A) as maximum during the disengagement of the clutch. At the end of disengagement, the currents raise up to 9(A) due to the performance of the motor which may lead to an error. Fig.7 (b) shows that currents go to 4.2(A) as maximum during the engagement of the clutch. At the end of the engagement of the clutch, the increase of currents is due to the stop of the movement of the driven part of the clutch. 


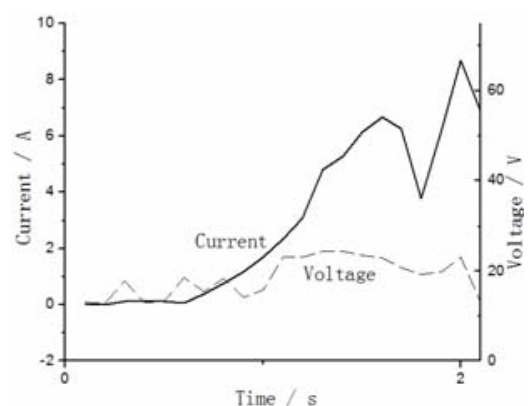

(a) Disengagement

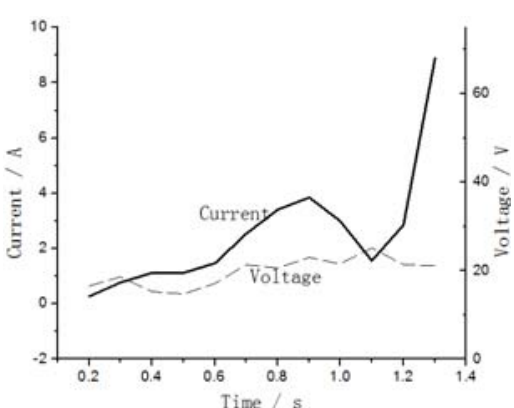

(b) Engagement

Fig.7 Current and voltage of the motor during action of the clutch

Sensors of engine speed, speed of the input shaft of the gearbox, and position of the driven part of the clutch indicate the dynamic characters of the powertrain. Rotational speeds and displacement of the driven part of the gearbox are depicted in Fig.8. The control algorithm profiles of the test are based on the simulations presented in the 4th part of this paper. The differences between the simulations and tests are the throttle of the engine, because of the engine speed is too high in the condition of full-throttle. Weighting factors of $\alpha$ and $\beta$ in the 3 rd part of the paper are defined to $(1,0)$ as the throttle is $5 \%$, and to $(0.6,0.4)$ as the throttle is $10 \%$ in the test.

The speed of the input shaft of the gearbox synchronizes the engine speed at $0.26 \mathrm{~s}$ when the throttle is $5 \%$ (Fig.8 (a)), while the time of synchronization is $0.15 \mathrm{~s}$ when the throttle is $10 \%$ (Fig.8 (c)). The clutch is forced to engage following the rule of 'fast-slow-fast' in the both two conditions (Fig.8 (b) \& (d)), which is similar to the control algorithm profile of the clutch on AMT.

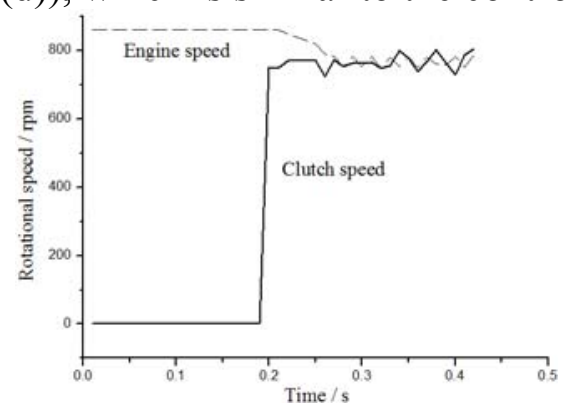

(a) Rotational speed at throttle of 5\%

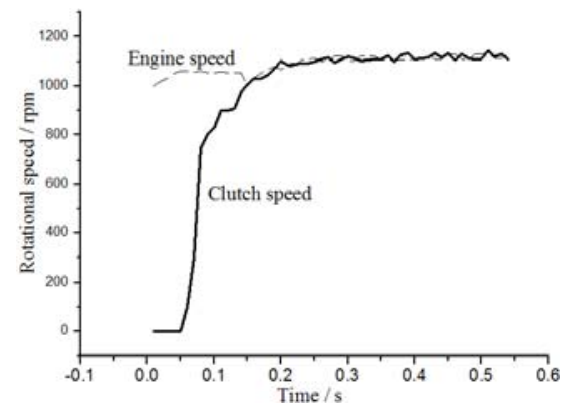

(c) Rotational speed at throttle of $10 \%$

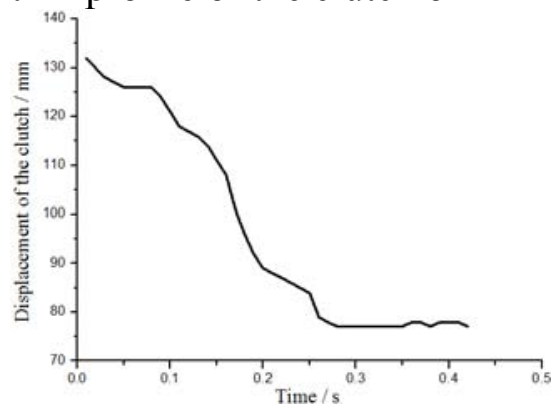

(b) Displacement at throttle of 5\%

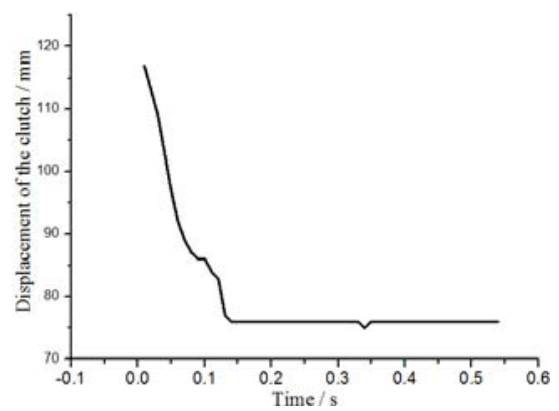

(d) Displacement at throttle of $10 \%$

Fig.8 Test results of the dynamic characters during launch

\section{Conclusions}

As a novel type of automatic transmission, OCT has the similar dynamic characters with that on AMT during launch. The dynamic model presented in this paper is appropriate to simulate the process of launch. The controller designed in the paper could control the engagement and disengagement of the clutch in launch conditions under different throttle angles. 


\section{References}

[1] Qin Datong. Universal Clutch Starting Control of AMT/DCT Automatic Transmission Based on Optimal Control. Journal of mechanical engineering, 2011, 47(12):85-91.

[2] Tongli Lu, Feng Dai, etc. Optimal control of dry clutch engagement based on the driver's starting intentions. Proc IMechE Part D: Journal of Automobile Engineering, 2012, 226(8):1048-1057.

[3] Joachim Horn, etc. Flatness-based clutch control for automated manual transmissions. Control Engineering Practice, 2003, 11: 1353-1359.

[4] Xingyong Song. Pressure-Based Clutch Control for Automotive Trans- missions using a Sliding-Mode Controller. IEEE/ASME Transactions on mechatronics, 2012, 17(3):534-546.

[5] Kong Huifang, Zhang Chongwei. A research of fuzzy control technology on AMT vehicle clutches. IEEE, 2006, 1_4244-03324/06.

[6] Chen Li, Dong YueHang, etc. System dynamic modeling and optimal torque control strategy for E.T. Driver based on AMT. WSEAS Transaction on Systems, 2008, 7(7): 742-757.

[7] Sun C S, Zhang Jianwu. Optimal control applied in automatic clutch engagement of vehicle. Journal of Mechanical Engineering, 2004, 17(2): 280-283.

[8] Amir Ibrahim, Qin Datong, etc. An improvement in the shift quality for Automatic Manual Transmission Using Multi-Variable Linear Quadratic Optimal Control Theory. Information Technology Journal, 2005, 4(3):239-245. 